\title{
A Catalog of Extragalactic Planetary Nebulae
}

\author{
G. H. Jacoby ${ }^{1}$ and A. Acker ${ }^{2}$ \\ ${ }^{1}$ WIYN Observatory, 950 N. Cherry Ave., Tucson, AZ 85712, USA \\ email: jacoby@wiyn.org \\ ${ }^{2}$ Observatoire de Strasbourg, 11, rue de l'Université, 7000 Strasbourg, FRANCE \\ email: acker@newb6.-u-strasbg.fr
}

\begin{abstract}
We are preparing a catalog of extragalactic planetary nebulae. The current number of entries is $\sim 8,000$ objects, with the largest samples coming from the Local Group (SMC, LMC, M33, and M31), but with representation from over 55 galaxies. The catalog is expected to be complete in late 2007.
\end{abstract}

Keywords. catalog, galaxies, SMC, LMC, M31

The discovery of new extragalactic planetary nebulae has been accelerating with the availability of large format CCD imagers and new instruments such as the PN Spectrograph (Douglas et al. (2002)). However, there is no catalog, as there is for Galactic PN (Acker et al. (1992)), to provide a uniform system for names and coordinates. The situation is nearly chaotic, with about 8,000 PNe now known in 55 galaxies and several galaxy clusters. The proposed extragalactic PN catalog serves a role that is analogous to the Galactic catalog, to bring order to current and future PN identifications.

About half of the samples come from the Local Group (SMC, LMC, M33, and M31), and at this time, the SMC catalog is complete, with a draft version available on-line at:

$$
\text { http://www.noao.edu/wiyn/jacoby/pncat/smc_pn.htm }
$$

The LMC catalog, along with most of the distant galaxies, will be completed in the summer of 2006 in collaboration with Quentin Parker. M31 will follow a year later.

The catalog uses a naming convention following IAU guidelines, and lists aliases to common names in the literature. Coordinates are given in J2000, along with: discoverer and the original coordinates, [OIII] and $\mathrm{H} \beta$ fluxes, systemic and expansion velocity, diameter, central star magnitude and type, and references in the literature. As an electronic catalog hosted by the Centre de Donnees Astronomiques de Strasbourg (CDS), new identifications can be added as they arise, and mis-identifications can be deleted easily. Observers are asked to send us their discoveries to be included in the catalog.

The selection of the most appropriate naming convention proved to be a controversial task. We adopted a naming convention of the form:

\section{PNE N3031 Jhhmmss.ss+ddmmss.ss}

and has the following positive features:

(a) it parallels the scheme used for the Galactic catalog (PN Glll.l+bb.b)

(b) it is consistent with IAU conventions

(c) it is self-documenting and unique; it derives from accurate epoch 2000 coordinates

(d) because names are unique, no catalog maintenance is required to extend or delete entries, and there are no collisions with new objects 
Lు

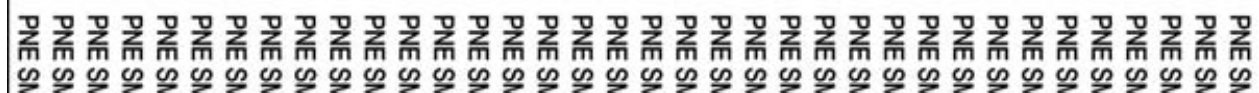

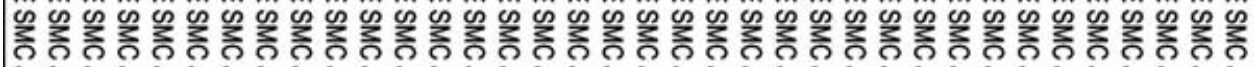

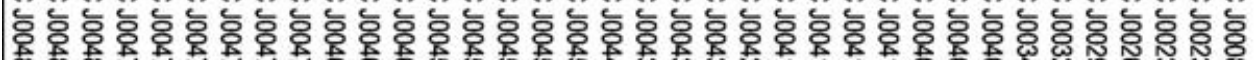

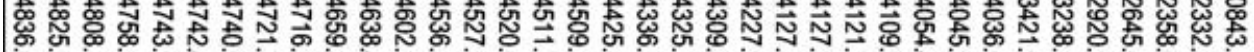

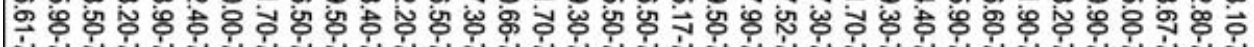

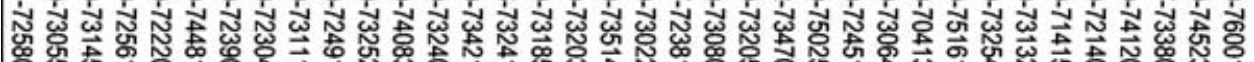

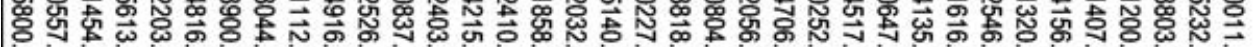
0

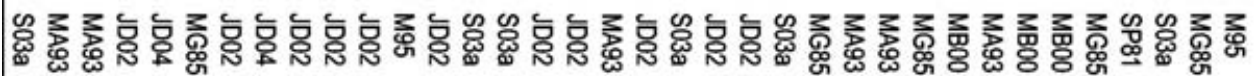

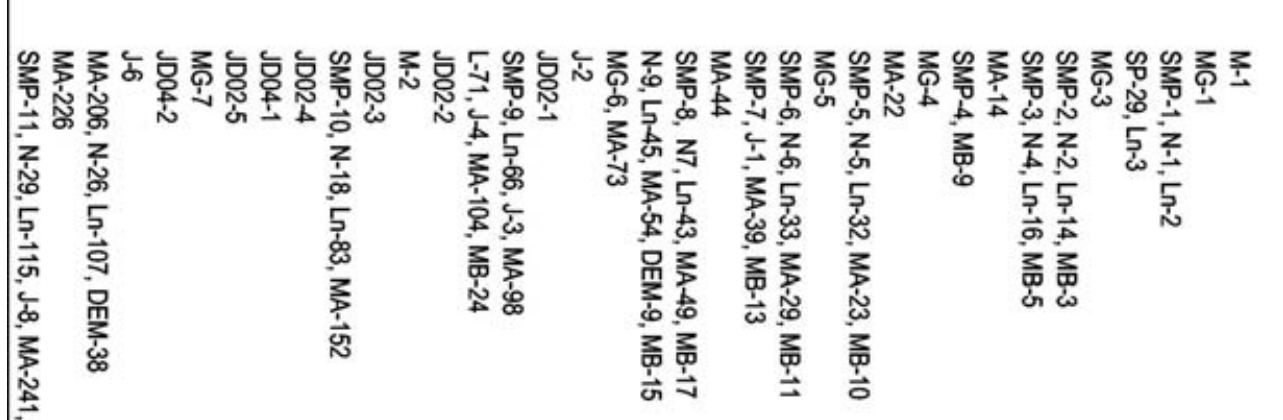

Figure 1. Sample of the first 36 entries in the SMC catalog with half the columns shown.

The disadvantage of the scheme is that names are lengthy and unattractive. The name may include the host galaxy when known, or, for intracluster PN, the galaxy cluster.

\section{References}

Acker, A., Marcout, J., Ochsenbein, F., Stenholm, B., \& Tylenda, R. 1992, Strasbourg - ESO catalogue of galactic planetary nebulae (Garching: ESO)

Douglas, N.G., Arnaboldi, M., Freeman, K.C., Kuijken, K., Merrifield, M.R., Romanowsky, A., Taylor, K., Capaccioli, M., Axelrod, T., \& Gilmozzi, R. 2002, PASP 114, 1234 